\title{
Children's Rehabilitation with Humanoid Robots and Wearable Inertial Measurement Units
}

\author{
Arzu Guneysu \\ Department of Computer Engineering \\ Bogazici University \\ Istanbul, Turkey \\ arzu.guneysu@boun.edu.tr
}

\author{
Bert Arnrich \\ Department of Computer Engineering \\ Bogazici University \\ Istanbul, Turkey \\ bert.arnrich@boun.edu.tr
}

\author{
Cem Ersoy \\ Department of Computer Engineering \\ Bogazici University \\ Istanbul, Turkey \\ cem.ersoy@boun.edu.tr
}

\begin{abstract}
The purpose of this work is to design a human computer interaction scheme for children in arm rehabilitation therapy. A humanoid robot demonstrates selected arm rehabilitation motions to children. Wearable inertial measurement units (IMUs) are used to monitor children's movements in order to give them proper feedback during therapy and to evaluate their motion performance. Preliminary experiments are done with 5 therapy motions which were selected by physiotherapists. Each motion is related to a goal based functional activity in order to help children to understand and perform the motion easily. Data from children and physiotherapists are recorded and analyzed. It is observed how children accept the robot and the IMUs. Preliminary results show various relationships between therapy motions and specific quaternion axes.
\end{abstract}

Keywords-Wearable Sensors, Human Robot Interaction, Rehabilitation

\section{INTRODUCTION}

Physical therapy sessions include repetitive actions and patients usually have to practice these actions regularly over weeks. Children often lose their interest in performing the tasks in the right way. A game-like child robot interaction design can increase the participation of the child to the therapy activity by increasing the motivation of the child [1].

Humanoid robots have been used for rehabilitation purposes by many researchers. Often, the aim is to enhance the ranges of motion and the use of the impaired limb in daily activity. Belpaeme et al. showed that physical robots can effectively grasp the attention of children and that adaptation to user characteristics can be a useful tool in supporting long term interaction [2]. The feasibility of utilizing a humanoid robot in rehabilitation is showed also by Choe et al. [3]. Fridin et al. proposed a human-robot interaction scheme with the humanoid Nao robot (see Figure 1) for children with Cerebral Palsy that motivates them to participate in the therapy activity [4].

In order to achieve interactive engagement that catches the child's attention and to motivate the child to imitate the robot in a rehabilitation session, the robot should recognize the actions of the child and provide feedback accordingly. Calculating similarity between the motions of the child and robot is crucial in order to provide a proper feedback in any physical therapy [5].

The main goal of this research effort is to contribute to the evolution of long-term child-robot interaction in an imitation game scenario within a physiotherapy session by automatically

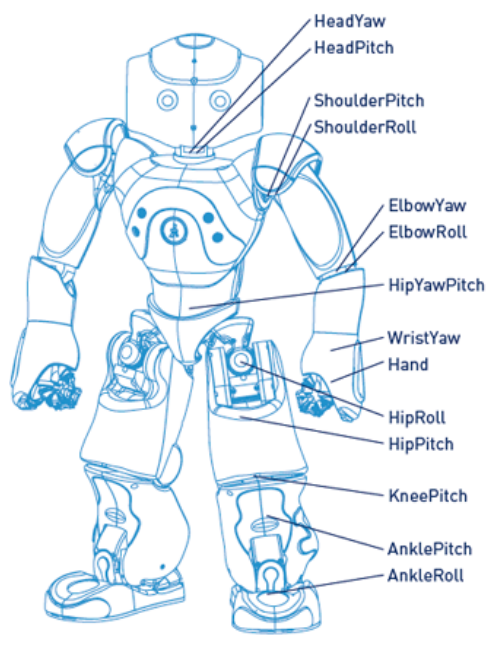

Fig. 1. Humanoid Robot Nao

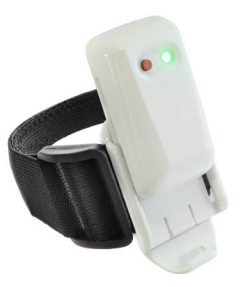

Fig. 2. EXL-S3 Wireless Inertial Measurement Unit

assessing the action performed by the child via wearable inertial sensor data. Our proposed robotic rehabilitation and evaluation system does not aim to substitute the physiotherapist, but to help in the therapeutic tasks.

\section{SYSTEM OVERVIEW}

Since imitation has a crucial role in child robot engagement, the proposed system includes a humanoid robot Nao that can perform arm rehabilitation activities such as vertical flexion easily. Nao is a programmable, $57 \mathrm{~cm}$ tall humanoid robot with 25 degrees of freedom (DOF) and its schematic diagram can be seen in Figure 1.

In order to implement an adaptive robot behavior that catches the child's attention and motivates the child to imitate the robot itself in a rehabilitation session, recognizing the 


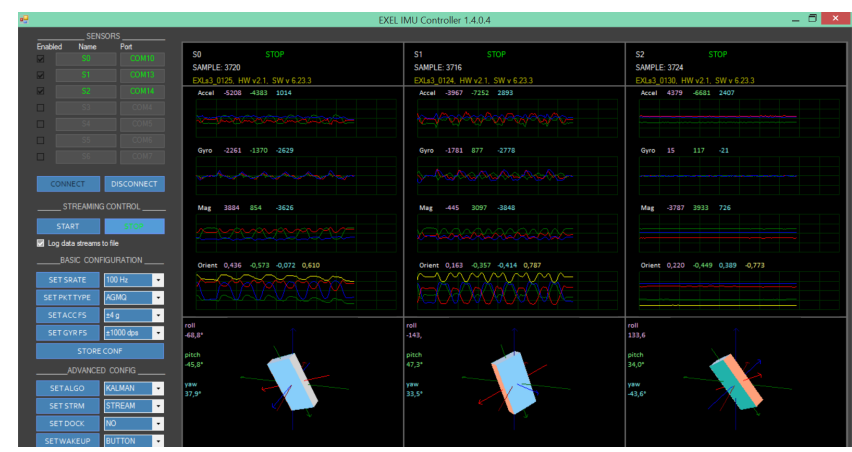

Fig. 3. EXEL IMU Controller Interface

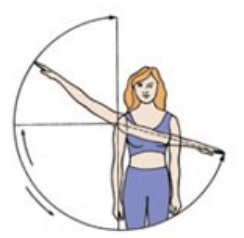

Abduction \& Adduction

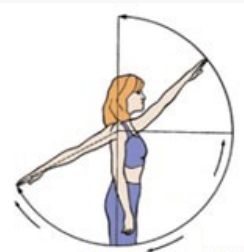

Flexion \& Extension

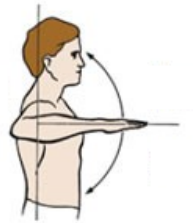

Internal \& External Rotation
Fig. 4. Physiotherapy motions of the arm

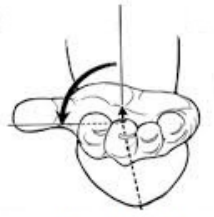

Supination

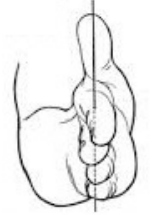

Neutral

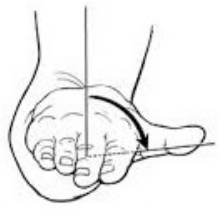

Pronation

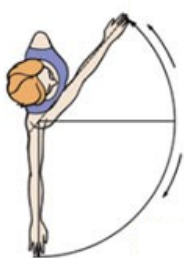

Horizontal Abduction \& Adduction
Fig. 5. Physiotherapy motions of the lower arm

imitation performance of the child is crucial. In our previous study we used the Kinect sensor to calculate angles of motions and we detected a limitation during experiments: The therapist stays in front of the children and gives them some objects or toys during a functional activity and this creates a barrier to Kinect vision. Therefore, we decided to use wireless inertial measurement units (IMUs) as an alternative way to monitor motions in real-time. We use the EXL-S3 wireless IMU modules from Exel (see Figure 2). The size of the module is $54 \mathrm{~mm} \times 33 \mathrm{~mm} \times 14 \mathrm{~mm}$ and it weighs $22 \mathrm{~g}$. It features tri-axial accelerometer, gyroscope and magnetometer; and it outputs its orientation in quaternion form where the estimation is done with Kalman filtering. Exel IMU Controller is used for data collection and each data sequence is visualized during the experiments; an example can be seen in Figure 3.

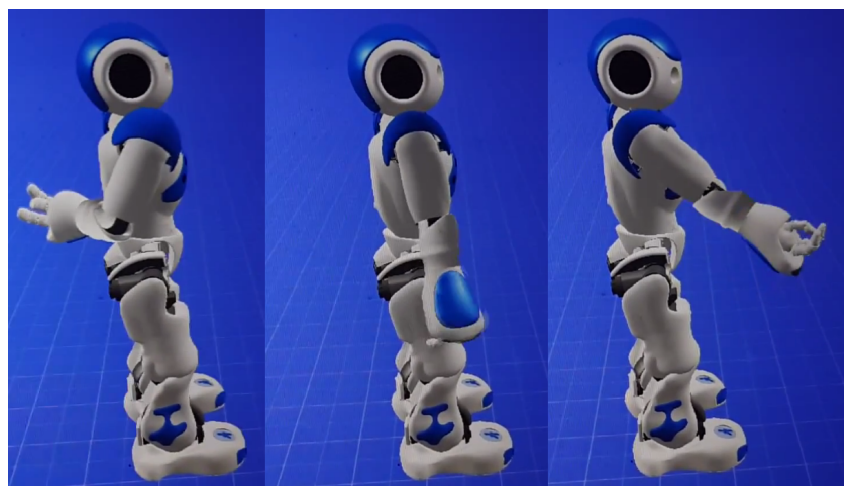

Fig. 6. 'Taking a pen from back' Action of Nao

\section{EXPERIMENTAL DESIGN}

\section{A. Activity Selection}

Our previous study was based on designing a physiotherapy session conducted with children under the guidance of a robot In the session, the robot performed the selected physiotherapy actions and the child imitated the robot. The success of imitation was measured by processing the skeleton joint angles of the child obtained by Kinect and grading the similarity [5]. Initial experiments were carried out with healthy children in an upper body physiotherapy imitation scenario. The results showed us that physiotherapy actions known from literature (as in Figure 4 and 5) can be misunderstood by the child. Such misunderstandings affect how the child performs the actions. In order to make it easier for the child to understand the physiotherapy actions, functional activities that include the desired therapy motions were selected together with the therapist. These activities are as follows:

- Opening a door with a key: It includes supination and pronation motions and the person rotates his/her hand like holding and turning a key.

- Touching the opposite shoulder with hand: It includes horizontal adduction and the person touches his/her left shoulder with the right arm without rotating the body.

- Taking an object from back of the neck: It includes external rotation and the person takes an imaginary object, such as a colorful pen, from the back of his/her neck.

- Taking an object from the back: It includes internal rotation and the person takes an imaginary object, such as a colorful pen, from his/her back.

- Reaching an object above the head: It includes vertical shoulder flexion and the person tries to reach an imaginary object, such as a colorful pen, above his/her head.

\section{B. Actions of Nao}

Selected functional actions were implemented on Nao via the Choreographe software [6] that lets users create and manage movements of the robot and determine the robot's joint angles for each motion. First, therapists were consulted 


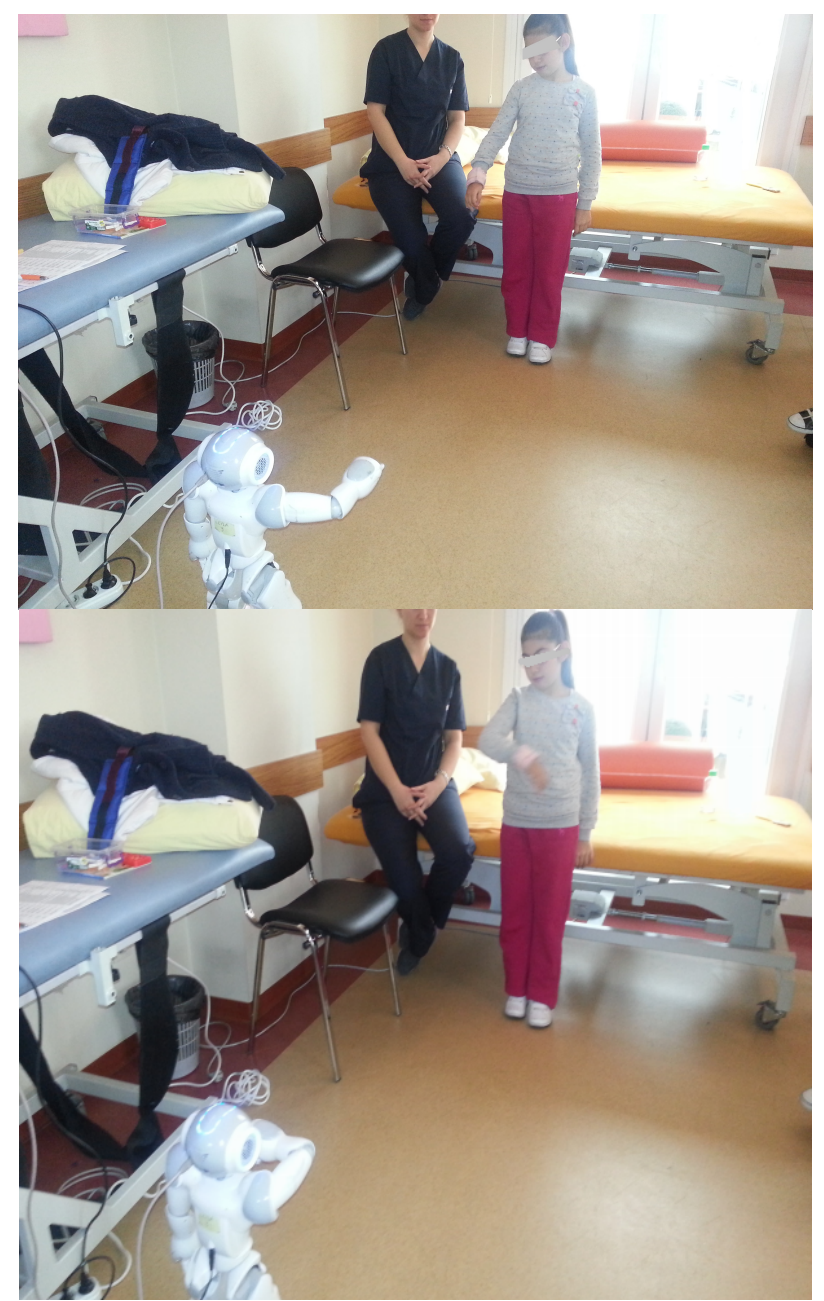

Fig. 7. Nao in The Therapy

and the simulated actions, such as the one shown in Figure 6 , were validated. Then, during the experiments, these preprogrammed actions were played.

\section{First Meeting with Nao}

In order to observe how children react to the Nao robot and to the wearable IMU modules, we introduced the system in a rehabilitation center for hemiparetic and diparetic children. Following ethical principals were applied:

- Proximity to the child shall not be in personal space at first but child can come closer if he/she desires

- Movements shall be smooth in order not to harm the child

- Children shall be asked if they would like to play with Nao or not

With these considerations, one 11 years old girl and one 5 years old girl experienced the system. An example scene can be seen in Figure 7. Both were observed to enjoy the robot. They wanted to do movements together and wanted the robot to imitate themselves. The younger child wanted the sensors to be removed after 5-6 minutes but the older one used them

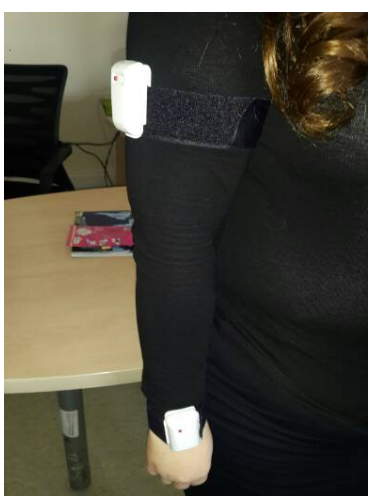

Fig. 8. Position of Sensors During Experiments

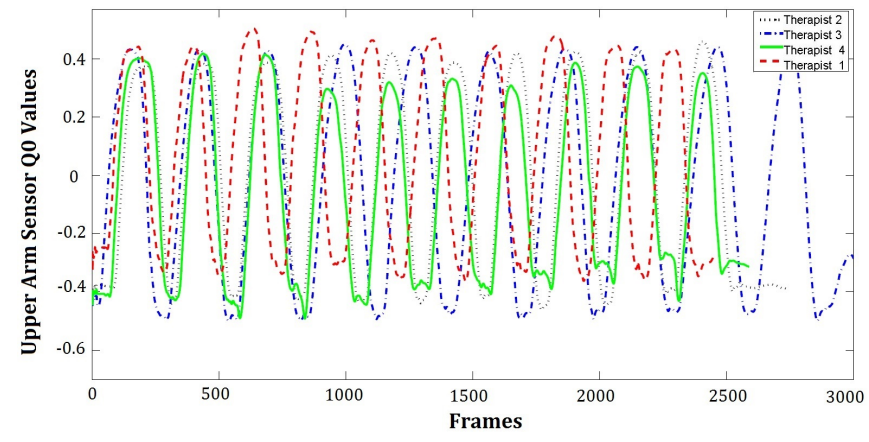

Fig. 9. Upper arm sensor, 3rd quaternion axis values of 10 sequential reaching actions of 4 therapists

throughout the session. We decided that we need a more appealing cover for the sensor to make the IMU modules more attractive for small children.

\section{Positioning of Sensors}

In the first experiments with IMUs worn by healthy children we collected data from 2 children by positioning two sensors aligned towards the posterior frontal plane. We chose a specific distance from the shoulder to place the upper arm sensor and a specific distance from the elbow to place the lower arm sensor. After examining the data of each functional activity, visualization of the data was not clear enough to distinguish actions, especially in the supination action where the child moves only the lower arm. Therefore, the position of the lower arm sensor was changed and the final positioning is shown in Figure 8.

\section{E. Capturing Therapist's Motion}

In addition to recording children's motion, we collected data from 4 physiotherapists. Each physiotherapist performed 5 selected functional actions 10 times and data was recorded by 2 sensors positioned as in Figure 8. They performed the actions in full range with proper angle values.

\section{F. Signal-based Statistical Features}

Before pre-processing, we visualized the data to ensure that the quaternion data of specific motions match as in Figure 9. After pre-proccessing, basic statistical features such as mean and variance were calculated since these features 


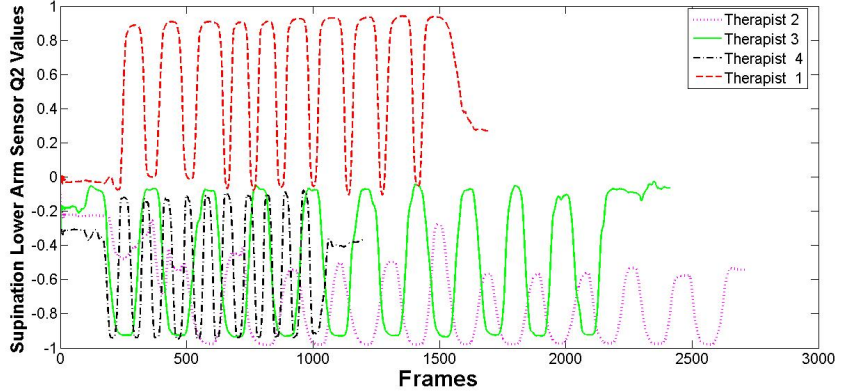

Fig. 10. Lower arm sensor, 2nd quaternion axis values of 10 sequential supination actions of 4 therapists

TABLE I. RELATIONSHIP BETWEEN ACTIONS AND QUATERNION AXIS DATA

\begin{tabular}{ccc}
\hline Action & Lower arm sensor & Upper arm sensor \\
\hline Key turn & 2. quat. axis & - \\
Touch shoulder & 1. quat. axis & 1. quat. axis \\
Take from neck & 2. 3. 4. quat. axes & 1. quat. axis \\
Take from back & 2. quat. axis & 1. 2. quat axes \\
Reach & 3.4. quat. axes & 3. 4. quat axes \\
\hline
\end{tabular}

provide high performance across a variety of activity recognition problems [7]. The features showed that physiotherapy actions have apparent relationship with specific quaternion axis data as outlined in the following and summarized in Table 1. For example, in the 'key turning' action data, we observed differences in means and found out that the initial position changes from person to person, affecting the mean (see Therapist 1 and Therapist 4 in Figure 10). During the experiment, Therapist 2 was observed to perform the motion with about half the amplitude (compared to other therapists). In Figure 10, the smaller peaks in the 2nd quaternion axis of Therapist 2 can be observed. This implies that there might be a useful relationship between standard deviation of the data and the turning amplitude of the arm.

\section{CONCLUSions}

In this study, a humanoid robot was used to increase the children's motivation in performing rehabilitation actions. As an alternative to Kinect, we used wireless IMUs for monitoring motion patterns. To eliminate mis-perception of complex motions, therapeutic motions were combined with functional activities. In preliminary experiments, we tested acceptance of the robot and IMUs, recorded preliminary data from healthy children and captured motion patterns from therapists. Experimental results indicated that there are relationships between specific quaternion axes and physiotherapy actions.

\section{ACKNOWLEDGEMENTS}

The authors thank Dilbade Rehabilitation Center physiotherapists for their collaboration and help. The research procedure and experiments were approved by the ethical committee of Bogazici University. This work was partially funded by the Co-Funded Brain Circulation Scheme Project "Pervasive Healthcare: Towards Computational Networked Life Science" (TUBITAK Co-Circ 2236, Grant agreement number: 112C005) supported by TUBITAK and EC FP7 Marie Curie Action COFUND.

\section{REFERENCES}

[1] D. A. Brooks and A. M. Howard, "Quantifying upper-arm rehabilitation metrics for children through interaction with a humanoid robot," Applied Bionics and Biomechanics, vol. 9, no. 2, pp. 157-172, 2012.

[2] T. Belpaeme, P. E. Baxter, R. Read, R. Wood, H. Cuayáhuitl, B. Kiefer, S. Racioppa, I. Kruijff-Korbayová, G. Athanasopoulos, V. Enescu et al., "Multimodal child-robot interaction: Building social bonds," Journal of Human-Robot Interaction, vol. 1, no. 2, pp. 33-53, 2012.

[3] Y.-k. Choe, H.-T. Jung, J. Baird, and R. A. Grupen, "Multidisciplinary stroke rehabilitation delivered by a humanoid robot: Interaction between speech and physical therapies," Aphasiology, vol. 27, no. 3, pp. 252-270, 2013.

[4] M. Fridin and M. Belokopytov, "Robotics agent coacher for cp motor function (rac cp fun)," Robotica, vol. 32, no. 08, pp. 1265-1279, 2014.

[5] A. Guneysu, R. D. Siyli, and A. A. Salah, "Auto-evaluation of motion imitation in a child-robot imitation game for upper arm rehabilitation," in Robot and Human Interactive Communication, 2014 RO-MAN: The 23rd IEEE International Symposium on. IEEE, 2014, pp. 199-204.

[6] E. Pot, J. Monceaux, R. Gelin, and B. Maisonnier, "Choregraphe: a graphical tool for humanoid robot programming," in Robot and Human Interactive Communication, 2009. RO-MAN 2009. The 18th IEEE International Symposium on. IEEE, 2009, pp. 46-51.

[7] A. Bulling, U. Blanke, and B. Schiele, "A tutorial on human activity recognition using body-worn inertial sensors," ACM Computing Surveys (CSUR), vol. 46, no. 3, p. 33, 2014. 